TRANSACTIONS OF THE

AMERICAN MATHEMATICAL SOCIETY

Volume 330, Number 2, April 1992

\title{
THE COMPLETE INTEGRAL CLOSURE OF $R[X]$
}

\author{
THOMAS G. LUCAS
}

\begin{abstract}
For a reduced ring $R$ that is completely integrally closed it is not always the case that the corresponding polynomial ring $R[X]$ is completely integrally closed. In this paper the question of when $R[X]$ is completely integrally closed is shown to be related to the question of when $R$ is completely integrally closed in $T(R[X])$ the total quotient ring of $R[X]$. A characterization of the complete integral closure of $R[X]$ is given in the main theorem and this result is used to characterize the complete integral closure of the semigroup ring $R[S]$ when $S$ is a torsion-free cancellative monoid.
\end{abstract}

\section{INTRODUCTION}

In what follows all rings are assumed to be commutative with nonzero unit and to contain no nonzero nilpotents. Unless otherwise specified, when we refer to the complete integral closure of a ring $R$ we mean the complete integral closure of $R$ in $T(R)$ the total quotient ring of $R$. The main objective of this paper is to determine when the polynomial ring $R[X]$ is completely integrally closed and to characterize its complete integral closure when it is not.

It is well known that for an integral domain $R, R$ is completely integrally closed if and only if the polynomial ring $R[X]$ is completely integrally closed. The same result does not hold for rings with zero divisors. If $R$ contains a nonzero nilpotent element $k$, then $(k / X)^{n}=0$ for some $n>1$. Hence in this case $R[X]$ is not integrally closed so it cannot be completely integrally closed. Thus we are left with considering what happens when $R$ is a reduced ring. Examples in [BCM, Lu1 and Q], the latter by way of [A1, Theorem 2.1], show that even if $R$ is a reduced total quotient ring, $R[X]$ need not be integrally closed so it need not be completely integrally closed. In our first example we construct a ring similar to that found in [Lu1, Example 3] with the exception that in this case $R[X]$ is integrally closed but not completely integrally closed even though again $R$ is its own total quotient ring.

Two rings which play important roles in determining when $R[X]$ is completely integrally closed are the complete ring of quotients $Q(R)$ and the ring of finite fractions $Q_{0}(R)$. Both can be realized as direct limits. For $Q(R)$ let $\mathscr{D}$ be the set of dense ideals of $R$; i.e., $\mathscr{D}=\{J: r J=(0)$ implies $r=0\}$. Then $Q(R)=\lim _{\longrightarrow}\{\operatorname{Hom}(J, R): J \in \mathscr{D}\}$. To form $Q_{0}(R)$, let $d$ be the set

Received by the editors October 15, 1989 and, in revised form, January 23, 1990.

1980 Mathematics Subject Classification (1985 Revision). Primary 13B20, 13 B25.

Key words and phrases. Almost integral, complete integral closure, complete ring of quotients, dense ideal. 
of those dense ideals of $R$ which contain finitely generated dense ideals of $R$. Then $Q_{0}(R)=\lim \{\operatorname{Hom}(J, R): j \in d\}$.

As we will see, both the complete integral closure and the integral closure of $R$ in $T(R[X])$ can also be constructed using direct limits. Underlying all of these constructions are a few basic facts about dense ideals and $R$-module homomorphisms defined on them.

Consider the construction of $Q_{0}(R)$ a la Lambek's construction of $Q(R)$ [Lam, Chapter 2].

Let $J_{1}$ and $J_{2}$ be finitely generated dense ideals of $R$ and let $f_{1} \in$ $\operatorname{Hom}\left(J_{1}, R\right), f_{2} \in \operatorname{Hom}\left(J_{2}, R\right)$. Then $J_{1} J_{2}$ is a finitely generated dense ideal of $R$ and $f_{1}+f_{2}$ and $f_{1} f_{2}$ make sense as elements of $\operatorname{Hom}\left(J_{1} J_{2}, R\right)$. The same definitions of addition and multiplication are used when $J_{1}$ and $J_{2}$ are ideals in the set $d$. Define $f_{1}$ and $f_{2}$ to be equivalent if they agree on some dense ideal $J$. For such a pair of homomorphisms and dense ideal $J$, let $a \in J_{1} J_{2}$ and $b \in J$. Since $f_{1}(b)=f_{2}(b)$ and $a$ is in both $J_{1}$ and $J_{2}$, $b f_{1}(a)=a f_{1}(b)=a f_{2}(b)=b f_{2}(a)$. As $J$ is dense, we must have $f_{1}(a)=f_{2}(a)$ for all $a$ in $J_{1} J_{2}$; in other words, $f_{1}$ and $f_{2}$ agree on some dense ideal of $R$ if and only if they agree on $J_{1} J_{2}$. The ring $Q_{0}(R)$ is formed from the equivalence classes of homomorphisms.

While it is not the case that an (completely) integrally closed ring is locally (completely) integrally closed, the converse is true. For if $t \in T(R)$ is in $R_{M}$ for each maximal ideal $M$, then the ideal $\left(R:_{R} t\right)$ is not contained in any maximal ideal of $R$. Whence $t \in R$. In the event that $R_{M}$ is an (completely) integrally closed domain for each maximal ideal $M$, then the polynomial ring $R[X]$ is (completely) integrally closed ring. Also, if $R$ sits well in a ring $Q$ in the sense that $T(R[X]) \subset T(Q[X])$ and $Q$ is locally an (completely) integrally closed domain, we can conclude that at least the (complete) integral closure of $R[X]$ is contained in the polynomial ring $Q[X]$. If $R$ contains nonzero nilpotents, such a ring $Q$ can never be found as the nilradical of $R$ must survive in some localization of $Q$. But when $R$ is reduced, there is a natural candidate for the ring $Q$, the complete ring of quotients $Q(R)$. For a reduced ring $R, Q(R)$ is von Neumann regular and every von Neumann regular ring is locally a field. Moreover, both $T(R)$ and $Q_{0}(R)$ sit naturally in $Q(R)$ and we can view $T(R[X])$ as a subring of $T(Q(R)[X])$. Hence both the integral closure and the complete integral closure of $R[X]$ are contained in $Q(R)[X]$.

Lemma 1 of [Lu2] shows how to view the elements of $Q_{0}(R)$ as quotients of polynomials in $T(R[X])$ so that $Q_{0}(R)[X]$ can be viewed as a subring of $T(R[X])$. We put this together with our Lemma 1.4 to show that if $b \in T(R[X])$ is almost integral over $R[X]$, then $b$ reduces to a polynomial over $Q(R)$ and hence to a polynomial over $Q_{0}(R)$. In Theorem 1.6 we show that the complete integral closure of $R[X]$ is the ring $R^{\#}[X]$ where $R^{\#}$ is the complete integral closure of $R$ in $T(R[X])$. Moreover, $R^{\#}=\underset{\longrightarrow}{\longrightarrow}\{\operatorname{Hom}(J, J): J \in d\}$ so $R^{\#} \subset Q_{0}(R)$. Note that this does not imply that $R^{\#}$ is the complete integral closure of $R$ in $Q_{0}(R)$.

Unlike integral closure, complete integral closure does not behave well with respect to taking (regular) quotient rings. For example, let $E$ be the ring of entire functions and let $S$ be the set of functions with at most finitely many (different) zeroes. It turns out that $E$ is completely integrally closed but $E_{S}$ 
is not [G1, Exercises 16 and 21, pp. 147 and 148]. Oddly enough the same sort of thing can happen between $R[X]$ and $T(R)[X]$. While $R[X]$ being integrally closed implies $T(R)[X]$ is integrally closed, it is possible for $R[X]$ to be completely integrally closed and $T(R)[X]$ to fail to be completely integrally closed. We present such a pair of rings in our Example 2.4.

In $\S 3$ we extend the main theorem of this paper as well as Theorem 3 of [Lu2] to semigroup rings. Specifically we consider semigroup rings of the form $R[S]$ where $S$ is a torsion-free cancellative abelian monoid with quotient group $G$. As in [G2] we denote the complete integral closure of $S$ in $G$ by $S^{*}$ and the integral closure of $S$ in $G$ by $S^{\prime}$ : by definition $S^{*}=\{t \in G$ : for some $s \in S$, $s+n t \in S$ for all $n \geq 1\}$ and $S^{\prime}=\{t \in G: n t \in S$ for some $n \geq 1\}$. As in the case of polynomial rings, we can view $Q_{0}(R)$ as a subring of $T(R[S])$. From this viewpoint the integral closure of $R$ in $T(R[S])$ is the same as the integral closure of $R$ in $Q_{0}(R)$. Denoting the integral closure of $R$ in $Q_{0}(R)$ by $R^{\ell}$ and using Chapter 12 of [G2] as a guide we show that $R^{\#}\left[S^{*}\right]$ is the complete integral closure of $R[S]$ and that $R^{\&}\left[S^{\prime}\right]$ is the integral closure of $R[S]$.

Any unexplained terminology or notation is standard as in [G1] and [Hu].

\section{THE COMPLETE INTEGRAL CLOSURE OF $R[X]$}

We begin with an example of a total quotient ring $R$ for which the polynomial ring $R[X]$ is integrally closed but not completely integrally closed.

Let $D$ be an integral domain and let $\mathscr{P}$ be a set of prime ideals of $D$ such that $\bigcap P_{a}=(0)$. Let $\mathscr{A}$ be an index set for $\mathscr{P}$ and let $\mathscr{I}=\mathscr{A} \times \mathbf{N}$ where $\mathbf{N}$ is the set of natural numbers. For each $i=(a, n)$ in $\mathscr{I}$, let $D_{i}=D / P_{a}$ and let $K_{i}=\mathrm{q} f\left(D_{i}\right)$. Construct the $D$-module $B=\sum K_{i}$ and form the ring $R=D+B$ from the direct sum of $D$ and $B$ by defining multiplication as $(r, b)(s, c)=(r s, r c+s b+b c)$. Here we are viewing $B$ both as a $D$-module and as a ring without unit.

For $r$ in $D$ and $i$ in $\mathscr{I}$, we denote the canonical image of $r$ in $K_{i}$ by $(r)_{i}$. Similarly for $b$ in $B$ we shall use $(b)_{i}$ to denote the $i$ th component of $b$. It is elementary to show that $(r, b)$ is a zero divisor if and only if $(r)_{i}=-(b)_{i}$ for some $i$ in $\mathscr{I}$. In particular, if $r \in \bigcup P_{a}$, then for all $b$ in $B,(r, b)$ is a zero divisor. Whence we can identify the total quotient ring of $R$ with $D_{S}+B$ where $S=D \backslash \bigcup P_{a}$.

For a finitely generated ideal $J=\left(\left(a_{1}, b_{1}\right),\left(a_{2}, b_{2}\right), \ldots,\left(a_{n}, b_{n}\right)\right), J$ has a nonzero annihilator if and only if for some $i \in \mathscr{I},\left(a_{j}\right)_{i}=-\left(b_{j}\right)_{i}$ for $j=1,2, \ldots, n$. In particular, if $\left(a_{1}, a_{2}, \ldots, a_{n}\right) \subset P_{a}$ for some $P_{a} \in \mathscr{P}$, then $J$ has a nonzero annihilator.

Example 1.1. Let $D=K\left[\left\{U Y^{n}: n \geq 0\right\},\left\{V Y^{n}: n \geq 0\right\}\right]$ and let $\mathscr{P}$ be the set of prime ideals of $D$ which do not contain both $U$ and $V$. Form the ring $R=D+B$. Then $R[X]$ is integrally closed since $D$ is integrally closed [Lu3, Theorem 1.3]. However, $R[X]$ is not completely integrally closed.

For ease of notation we shall use lower case letters to denote $U, V$ and $Y$ as elements of $R$ and continue to use upper case when we consider them in $D$.

Consider the quotient of polynomials $b=(u y X+v y) /(u X+v)$. Since $(U, V)$ is not contained in any $P_{a}, u X+v$ is not a zero divisor of $R[X]$. Hence $b$ is in $T(R[X]) \backslash R$. Essentially $b$ represents $Y$ as an element of $T(R[X])$ but since $Y$ is not in $D, y$ is not an element of $R$. As an element 
of the quotient field of $D, Y$ is almost integral over $D$ as $U Y^{n}$ and $V Y^{n}$ are in $D$ by definition. Whence, $b^{n}(u X+v)$ is in $R[X]$ for all $n$ and since $u X+v$ is not a zero divisor, $b$ is almost integral over $R[X]$. However, even more is true. Since $b^{n} u=y^{n} u$ and $b^{n} v=y^{n} v$ are in $R, b^{n}$ is in the finitely generated $R$-module $M=(X /(u X+v)) R+(1 /(u X+v)) R$. Hence $b$ is almost integral over $R$ (as an element of $T(R[X])$ ). Also multiplication by $b$ defines an $R$-module endomorphism on the dense ideal $J$ generated by the set $\left\{y^{n} u, y^{n} v: n \geq 0\right\}$.

Recall that for a polynomial $g(X) \in R[X]$, the ideal of $R$ generated by the coefficients of $g$ is called the content of $g$. We use $c(g)$ to denote this ideal.

Proofs of the following two lemmas can be found in [Lu2].

Lemma 1.2 (cf. [Lu2, Lemma 1]). Let $R$ be a reduced ring and let $s$ be in $Q_{0}(R)$. Then there exist polynomials $f(X)$ and $g(X)$ in $R[X]$ with $c(g)$ dense in $R$ such that $s \in \operatorname{Hom}(c(g), R)$ and for each $j, s\left(g_{j}\right)=f_{j}$. In particular, $Q_{0}(R)[X]$ can be considered as a subring of $T(R[X])$.

Lemma 1.3 (cf. [Lu2, Lemma 2]). Let $R$ be a reduced ring and let $s \in Q(R)$. If $s J \subset Q_{0}(R)$ for some finitely generated dense ideal $J$ of $R$, then $S \in Q_{0}(R)$.

Before presenting our characterization of the complete integral closure of $R[X]$, we need two more lemmas.

Lemma 1.4. Let $R$ be a reduced ring. If $s(X)$ is in $Q(R)[X] \cap T(R)[X]$, then $s(X)$ is in $Q_{0}(R)[X]$.

Proof. Let $s(X) \in Q(R)[X] \cap T(R[X])$. Then $s(X)=f(X) / g(X)$ for some $f(X)$ and $g(X)$ in $R[X]$. If $s(X)$ is not in $Q_{0}(R)[X]$, we may assume it is of minimal degree. Write $s(X)=s_{k} X^{k}+\cdots+s_{0}$ and $g(X)=g_{m} X^{m}+\cdots+g_{0}$. By Lemma 1.3, we may assume $k>0$. By Lemma 1.2 , if $s_{k}$ is in $Q_{0}$, then $s(X)-s_{k} X^{k}$ is in $Q(R)[X] \cap T(R[X])$ contradicting the minimality of degree of $s(X)$. Thus $s_{k} \in Q(R) \backslash Q_{0}(R)$. However $s_{k} g_{m}$ is in $R$ so again by the minimality of degree of $s(X)$ it must be that $g_{m} s(X)$ is a polynomial in $Q_{0}(R)[X]$. In particular, $s_{k-1} g_{m}$ is in $Q_{0}(R)$ so that $s_{k} g_{m-1}$ must also be in $Q_{0}(R)$ since the sum of the two is in $R$. Thus by minimality, $g_{m-1} s(X)$ is a polynomial in $Q_{0}(R)[X]$. Inductively we get $g_{j} s(X) \in Q_{0}(R)[X]$ for $j=$ $m, m-1, \ldots, 0$. In particular, $s_{k} g_{j}$ is in $Q_{0}(R)$ for all $j$ which by Lemma 1.3 implies $s_{k} \in Q_{0}$ contradicting the minimality of degree of $s(X)$. Therefore, $s(X) \in Q_{0}(R)[X]$.

Using the above lemmas together with the fact that $Q(R)[X]$ is completely integrally closed we may conclude that the complete integral closure of $R[X]$ is a subring of $Q_{0}(R)[X]$.

Lemma 1.5. Let $R$ be a reduced ring and let $s(X) \in Q_{0}(R)[X]$ be almost integral over $R[X]$ as an element of $T(R[X])$. If $g(X) \in R[X]$ is a polynomial of degree $m$ such that $s_{k} g_{j} \in R$ for all $k$ and $j$ and $s(X)^{n} g(X)$ is in $R[X]$ for all $n$, then $s_{0}^{n} g_{j} g_{k}^{j} \in R$ for all $j=0, \ldots, m, k=j, j+1, \ldots, m$ and all $n \geq 1$.

Proof. Clearly, $s_{0}^{n} g_{0} \in R$ for all $n$.

For $p$ between 1 and $m$ consider $A_{p}$, the coefficient on $X^{p}$ in the expansion of $s(X)^{n} g(X)$. for $p=1$ we have $A_{1}=s_{0}^{n} g_{1}+n s_{1} s_{0}^{n-1} g_{0}$. As $s_{0}^{n} g_{0}$ is in $R$ for all $n$ and $s_{k} g_{j}$ is in $R$ for all $k$ and $j$, if we multiply through by $g_{j}$ we see that $s_{0}^{n} g_{1} g_{j}$ is in $R$ for all $n$. 
Assume the result holds for $p=0,1, \ldots, j-1$. For $p=j$, let $H_{t} s_{0}^{n-j+1}$ denote the coefficient on $X^{j-t}$ in the expansion of $s(X)^{n}$ and note that $H_{t}$ is a sum of products of $j-t s_{q}$ 's. As with $A_{1}$ multiply $A_{j}=s_{0}^{n} g_{j}+n s_{1} s_{0}^{n-1} g_{j-1}+$ $\cdots+H_{0} s_{0}^{n-j} g_{0}$ by $g_{k}^{j}$. As each $H_{t}$ is a sum of products of $j-t s_{q}$ 's $H_{t} g_{k}^{j-t}$ is in $R$ and by the induction step $s_{0}^{n-j+t} g_{t} g_{k}^{t}$ is also in $R$. Therefore since $A_{i}$ is in $R, s_{0}^{n} g_{j} g_{k}^{j} \in R$ for $k=j, j+1, \ldots, m$ and all $n \geq 1$.

Theorem 1.6. Let $R$ be a reduced ring. Then

(1) The complete integral closure of $R$ in $T(R[X])$ is the ring

$$
R^{\#}=\underset{\lim }{\longrightarrow}\{\operatorname{Hom}(J, J): J \in d\} \text {. }
$$

(2) The complete integral closure of $R[X]$ is $R^{\#}[X]$. In particular, $R[X]$ is completely integrally closed if and only if $R$ is completely integrally closed in $T(R[X])$.

Proof. Let $R^{\#}=\lim _{\longrightarrow}\{\operatorname{Hom}(J, J): J \in d\}$ and let $h \in R^{\#}$. Let $J \in d$ be such that $h J \subset J$ and let $\left(a_{0}, a_{1}, \ldots, a_{m}\right)$ be a dense ideal of $R$ contained in $J$. Then for all $n \geq 1$ and $j=0,1, \ldots, m, h^{n} a_{j} \in J$.

Consider the polynomial $a(X)=a_{m} X^{m}+\cdots+a_{0}$. Since $A$ is a dense ideal of $R, a(X)$ is not a zero divisor. To see that $h$ is almost integral over $R$ consider the $R$-module $\mathbf{M}=\left(X^{m} / a(X)\right) R+\left(X^{m-1} / a(X)\right) R+\cdots+(1 / a(X)) R$. Since $h^{n} a_{j}=b_{n, j} \in R$, we can write $h^{n}=\left(b_{n, m} X^{m}+\cdots+b_{n, 0}\right) / a(X) \in \mathbf{M}$. Hence $h$ is almost integral over $R$ (as an element of $T(R[X])$ ). Thus the complete integral closure of $R[X]$ contains $R^{\#}[X]$.

Now suppose that $h \in T(R[X])$ is almost integral over $R$. As $T(Q(R)[X]) \supset$ $T(R[X])$ and $Q(R)$ is von Neumann regular, $h \in Q(R)$. Whence by Lemma 1.4, $h \in Q_{0}(R)$. Let $g$ be a regular element of $R[X]$ such that $h^{n} g \in R[X]$ for all $n$. Then $c(g)$ is dense and $h \in \operatorname{Hom}(c(g), R)$. Let $J_{n}=\left\{r \in R: h^{n} r \in R\right\}$; $J_{n}$ is the largest dense ideal on which $h^{n}$ is defined. Since $c(g) \subset J_{n}$ for all $n, J=\bigcap J_{n}$ is dense and contains $c(g)$. Let $r \in J$. Then $h^{n} r \in R$ for all $n \geq 0$. In particular, for each $n \geq 1, h^{n+1} r=h^{n}(h r) \in R$ so $h r \in J_{n}$. Hence $h J \subset J$. Therefore the complete integral closure of $R$ in $T(R[X])$ is $R^{\#}=\lim _{\longrightarrow}\{\operatorname{Hom}(J, J): J \in d\}$.

Let $\vec{b}=f / g$ be almost integral over $R[X]$ with $b^{n} g \in R[X]$ for all $n$. Then $b=h(X) \in Q_{0}(R)[X]$ since $Q(R)[X]$ is completely integrally closed. Write $h(X)=h_{p} X^{p}+\cdots+h_{0}$ and $g(X)=g_{m} X^{m}+\cdots+g_{0}$. In the domain case we have $h_{p}^{n} g_{m}$ in $R$ for all $n$ so $h_{p}$ is almost integral over $R$. With rings containing zero divisors this is not enough since $g_{m}$ may be a zero divisor (even though $g(X)$ is not). However since a product of dense ideals is dense we may further assume that each of the products $h_{k} g_{j}$ is in $R$. Thus by Lemma 1.5, $h_{0}^{n} g_{j}^{j+1}$ is in $R$ for $j=0,1, \ldots, m$ and all $n \geq 1$. As $c(g)$ is a finitely generated dense ideal of $R$ so is $\left(g_{0}, g_{1}^{2}, \ldots, g_{m}^{m+1}\right)$. Thus $h_{0} \in R^{\#}$. Whence, $h(X)-h_{0}$ is almost integral over $R[X]$ and thus so is $\left(h(X)-h_{0}\right) X^{-1}$. By way of Lemma 1.5 and induction, we have $h_{k} \in R^{\#}$ for all $k$. Therefore $R^{\#}[X]$ is the complete integral closure of $R[X]$.

If $J$ is a regular ideal of $R$ and $h$ is an $R$-module homomorphism on $J$, then $h$ can be considered as an element of $T(R)$. For if $r$ is a regular element of $J$ with $h(r)=s$, then, as in the domain case, we can simply consider $h$ 
to be equal to multiplication by $s / r$. Thus if the only finitely generated dense ideals of $R$ are the ones which contain regular elements, we have that both $T(R)$ and $T(R)[X]$ are completely integrally closed in $T(R[X])$. A ring is said to have property A if every finitely generated ideal containing only zero divisors has a nonzero annihilator.

We shall find the following results useful when we consider complete integral closure in semigroups rings.

Corollary 1.7. Let $R$ be a reduced ring. Then the complete integral closure of $R\left[X, X^{-1}\right]$ is $R^{\#}\left[X, X^{-1}\right]$.

Proof. Since $Q(R)$ is locally a field, $Q(R)\left[X, X^{-1}\right]$ is completely integrally closed. As in Lemma 1.4, if $s \in T(R[X])$ is almost integral over $R\left[X, X^{-1}\right]$, then $s$ is in $Q_{0}(R)\left[X, X^{-1}\right]$. Let $g \in R\left[X, X^{-1}\right]$ be such that $s^{n} g$ is in $R\left[X, X^{-1}\right]$ for all $n$. By multiplying each $s^{n} g$ by a sufficiently large power of $X$, we will get the same relations used to establish Lemma 1.5. As in that lemma, an induction proof leads to the conclusion that $R^{\#}\left[X, X^{-1}\right]$ is the complete integral closure of $R\left[X, X^{-1}\right]$.

Corollary 1.8 (cf. [A1, Theorem 3.2]). Let $R$ be a reduced ring with property A. Then the complete integral closure of $R[X]$ is the ring $R^{*}[X]$ where $R^{*}$ is the complete integral closure of $R$ in $T(R)$. In particular, $R[X]$ is completely integrally closed if and only if $R$ is completely integrally closed.

Corollary 1.9. Let $R$ be a reduced ring and let $X_{1}, X_{2}, \ldots, X_{n}$ be indeterminates. Then the complete integral closure of $R\left[X_{1}, X_{2}, \ldots, X_{n}\right]$ is $R^{\#}\left[X_{1}, \ldots, X_{n}\right]$ and $R^{\#}\left[X_{1}, \ldots, X_{n}\right]\left[X_{1}^{-1}, \ldots, X_{n}^{-1}\right]$ is the complete integral closure of $R\left[X_{1}, \ldots, X_{n}\right]\left[X_{1}^{-1}, \ldots, X_{n}^{-1}\right]$.

Proof. For any ring $T$, the polynomial ring $T[X]$ has property A. Thus the complete integral closure of $R\left[X_{1}, X_{2}\right]$ is $R^{\#}\left[X_{1}, X_{2}\right]$ since $R^{\#}\left[X_{1}\right]$ is the complete integral closure of $R\left[X_{1}\right]$. Hence by induction $R^{\#}\left[X_{1}, \ldots, X_{n}\right]$ is the complete integral closure of $R\left[X_{1}, \ldots, X_{n}\right]$. Similarly, the complete integral closure of $R\left[X_{1}, \ldots, X_{n}\right]\left[X_{1}^{-1}, \ldots, X_{n}^{-1}\right]$ is

$$
R^{\#}\left[X_{1}, \ldots, X_{n}\right]\left[X_{1}^{-1}, \ldots, X_{n}^{-1}\right] \text {. }
$$

\section{RINGS OF THE FORM $R=D+B$}

If $R$ is a ring of the form $D+B$, then $Q(R)$ can be identified with the direct product $\prod K_{i}$. This is due to the facts that since $\cap P_{a}=(0), D$ can be considered as a subring of $\prod K_{i}$ and $B=\sum K_{i}$ is itself a dense ideal of $R$. In fact an alternate way to construct the ring $R$ is as the subring of $\prod K_{i}$ generated by $B$ and the canonical image of $D$ in $\prod K_{i}$ (for more on this method see [Hu, $\S 26]$ or [Lu3]). With this alternate view of $R$ it is easy to see that for a dense ideal $J$ of $R$, every $R$-module homomorphism is defined by multiplication by an element of $\prod K_{i}$. Thus if $(r, b) \in J$ such that $r \in S=R \backslash \bigcup P_{a}$, then $J$ is a regular ideal and so every homomorphism on $J$ can be defined by multiplication by an element of the form $(s / r, c) \in T(R)$-recall that we have identified $T(R)$ with $D_{S}+B$.

By Theorem 1.6, if $s \in T(R[X])$ is almost integral over $R$, then there exists an ideal $J$ containing a finitely generated dense ideal such that $s \in \operatorname{Hom}(J, J)$. 
Before we give a description of the complete integral closure of $R[X]$ for $R=$ $D+B$, we need the following lemma.

Lemma 2.1. Let $R=D+B$ and let $J \in d$. Then the set $J^{\prime}=\{a \in D:(a, b) \in$ $J$ for some $b \in B\}$ is an ideal of $D$ and $J=J^{\prime}+B$.

Proof. For an ideal $L$ of $R, L^{\prime}=\{a \in D:(a, b) \in L$ for some $b \in B\}$ is an ideal of $D$. This follows easily from the definition of multiplication on $R$. Hence $J^{\prime}$ is an ideal of $D$.

Since $J \in d$ there is a finitely generated dense ideal $A=\left(\left(a_{1}, b_{1}\right),\left(a_{2}, b_{2}\right)\right.$, $\left.\ldots,\left(a_{m}, b_{m}\right)\right)$ contained in $J$. Since $A$ is dense it must be that $A^{\prime}=\left(a_{1}, a_{2}\right.$, $\left.\ldots, a_{m}\right)$ is an ideal of $D$ which is not contained in any $P_{a}$. Thus for each $i \in \mathscr{I},\left(a_{j}\right)_{i}$ is not zero for some $j$. Since each $K_{i}$ is a field, $\left(a_{j}\right)_{i}$ is invertible. For lack of better notation we let $\left(a_{j}\right)_{i}^{-1}$ denote both the inverse of $\left(a_{j}\right)_{i}$ as an element of $K_{i}$ and the element of $B$ all of whose components are zero except the $i$ th one which is $\left(a_{j}\right)_{i}^{-1}$. Now multiply $\left(a_{j}, b_{j}\right)$ by $\left(0,\left(a_{j}\right)_{i}^{-1}\right)$ to get $\left(0,(1)_{i}\right)$. With $\left(0,(1)_{i}\right)$ in $J$ for each $i$, we have $B$ contained in $J$. Hence $J=J^{\prime}+B$.

Theorem 2.2. Let $R$ be a ring of the form $D+B$ and let $d^{\prime}$ denote the set of ideals of $D$ which contain a finitely generated ideal not contained in any $P_{a}$. Then the complete integral closure of $R$ in $T(R[X])$ can be identified with $D^{\#}+B$ where $D^{\#}=\bigcup\left\{\left(J^{\prime}: J^{\prime}\right): J^{\prime} \in d^{\prime}\right\}$.

Proof. Let $s^{\prime} \in D^{\#}$ and let $J^{\prime} \in d^{\prime}$ be such that $s^{\prime} J^{\prime} \subset J^{\prime}$. Then there is a finitely generated ideal $A=\left(a_{0}, a_{1}, \ldots, a_{m}\right) \subset J^{\prime}$ such that $A$ is not contained in any $P_{a}$. Since $s^{\prime} J^{\prime} \subset J^{\prime}, s^{\prime n} a_{j} \in D$ for $n \geq 0$. As a polynomial over $R$, $a(X)=a_{m} X^{m}+\cdots+a_{0}$ is a regular element of $R[X]$. Hence as in Theorem 1.6 we can define a homomorphism $s$ on $J=J^{\prime}+B$ by multiplication by the quotient $\left(s^{\prime} a_{m} X^{m}+\cdots+s^{\prime} a_{0}\right) / a(X)$. This gives us an element of $T(R[X])$ which is almost integral over $R$. Whence for any $b \in B,\left(s^{\prime}, b\right)$ is almost integral over $R$ as an element of $T(R[X])$.

Note that we have shown that multiplication by $s^{\prime}$ makes sense in $B$.

Let $s \in R^{\#}$ and let $J \in d$ be such that $s J \subset J$. Let $\left(\left(a_{1}, b_{1}\right),\left(a_{2}, b_{2}\right), \ldots\right.$, $\left.\left(a_{n}, b_{n}\right)\right)$ be a finitely generated dense ideal contained in $J$. By Lemma 2.1, $\left.A+B=\left(\left(a_{1}, 0\right)\right), \ldots,\left(a_{n}, 0\right)\right)$ and $J=J^{\prime}+B$ where $J^{\prime}=\{a \in D:(a, b) \in J$ for some $b \in B\}$ is in $d^{\prime}$. Define a map $s^{\prime}: J^{\prime} \rightarrow D$ by $s^{\prime}(a)=\varphi(s(a, 0))$ where $\varphi$ is the natural map from $R$ to $D$ defined by $\varphi(r, b)=r$. By the discussion preceding the theorem we know that $s$ can be defined by multiplication by an element of $\prod K_{i}$. As such it easy to see that $s(0, b) \in B$ for all $b \in B$. Hence $\varphi(s(a, 0))=\varphi(s(a, b))$ for all $b \in B$. Since $s^{\prime}$ is a $D$-module homomorphism on an ideal of $D$ it can be defined by multiplication by an element of $J^{\prime-1}$. Let $t \in J^{\prime-1}$ be such that $s^{\prime}(a)=t a$. As $s J \subset J$, we must have $s^{\prime} J^{\prime} \subset J^{\prime}$. Hence $t \in\left(J^{\prime}: J^{\prime}\right)$ so as above $t$ can be considered as an element of $T(R[X])$ which is almost integral over $R$. Thus we can consider $t$ to be an element of $\prod K_{i}$. Moreover, since $J=J^{\prime}+B$ and $J^{\prime}$ generates $J$ as an ideal of $R$ it must be that $s-t=c$ is an element of $B$. Therefore we can view $s$ as multiplication by $(t, c) \in D^{\#}+B$ and hence $R^{\#}=D^{\#}+B$.

Corlary 2.3. If $D$ is completely integrally closed and $\mathscr{P}$ is any set of prime ideals such that $\cap P_{a}=(0)$, then the corresponding ring $R=D+B$ is completely integrally closed in $T(R[X])$ and $R[X]$ is completely integrally closed. 
In our next example we construct a reduced ring $R=D+B$ with the property that $R[X]$ is completely integrally closed but $T(R)[X]$ is not. As in our first example we use upper and lower case letters to distinguish between the indeterminates used in defining $D$ and the image of these elements in the ring $D+B$.

Let $\left\{U_{j}\right\},\left\{V_{j}\right\}$ and $\left\{W_{j}\right\}$ be countably infinite sets of indeterminates and for each $j$ let $Z_{j}=U_{j} V_{j} / W_{j}$. Let $\mathbf{M}=\left\{\left(m_{1}, m_{2}, \ldots,\right): m_{j} \geq 0\right.$ for all $j\}$. For $m \in \mathbf{M}$, let $U^{m}$ denote the product $U_{1}^{m_{1}} U_{2}^{m_{2}} \cdots$ and define $V^{m}$, $W^{m}$ and $Z^{m}$ similarly. Let $\mathscr{U}=\left\{U^{m}: m \in \mathbf{M}\right\}, \mathscr{V}=\left\{V^{m}: m \in \mathbf{M}\right\}$, $\mathscr{W}=\left\{W^{m}: m \in \mathbf{M}\right\}$ and $\mathscr{Z}=\left\{Z_{m}: m \in \mathbf{M}\right\}$. In terms of elements of $\mathbf{M}$ we have $Z^{m}=U^{m} V^{m} / W^{m}$. Similar expressions hold for $U^{m}, V^{m}$ and $W^{m}$. Let $\mathbf{F}=\left\{m \in \mathbf{M}\right.$ : for some $n>0, m_{j}>0$ for all $\left.j \geq n\right\}$ and let $S=\left\{U^{m} V^{r} W^{s} Z^{t}: m, r, s, t \in \mathbf{F}\right\}$. Except for allowing infinite products of powers of indeterminates, our construction of $D$ is essentially the same as that used by Claborn to prove that every abelian group is the class group for some domain [C, Proposition 6].

Example 2.4 (cf. [C, Proposition 6]). Let $D=K[\mathscr{U}, \mathscr{V}, \mathscr{W}, \mathscr{Z}$ ] and let $\mathscr{P}$ be the set of prime ideals which miss $S$ and either $\mathscr{V}$ or $\mathscr{W}$. As in Claborn's example, $D$ is completely integrally closed. Hence the ring $R=D+B$ is such that $R[X]$ is completely integrally closed. However the same is not true for $T(R)$.

Consider the element $b=\left(Z_{1} Z_{2} \ldots\right) /\left(V_{1} V_{2} \ldots\right)=\left(U_{1} U_{2} \ldots\right) /\left(W_{1} W_{2} \ldots\right)$, $b$ is not in $D_{S}$ but $b$ is almost integral over $D_{S}$. In particular for $m=$ $(1,2, \ldots), b^{n} V^{m}=\left(Z_{1} Z_{2} \ldots\right)^{n}\left(V_{n+1} V_{n+2}^{2} \ldots\right) / V_{1}^{n-1} V_{2}^{n-2} \ldots V_{n-1} \in D_{S}$ for all $n \geq 1$. The expression for $b^{n} W_{m}$ is similar so we also have $b^{n} W^{m} \in$ $D_{S}$ for all $n \geq 1$. By our choice of prime ideals for the set $\mathscr{P}$, the finitely generated ideal $\left(V^{m}, W^{m}\right)$ is not contained in any $P_{a}$. Hence in $T(R[X])$ we can view $b$ as the quotient of polynomials $\left(z^{m} X+u^{m}\right) /\left(v^{m} X+w^{m}\right)$ and we have $b^{n} \in\left(X /\left(v^{m} X+w^{n}\right)\right) T(R)+\left(1 /\left(v^{m} X+w^{m}\right)\right) T(R)$ for all $n$. Thus $T(R)[X]$ is not completely integrally closed in $T(R[X])$.

In our next example we start with a ring $R=D+B$ that is completely integrally clcsed and show that it is possible that the complete integral closure of $R[X]$ is not completely integrally closed.

Example 2.5 (cf. [GH, Example 1]). Let $D=K\left[\left\{U^{2 n+1} W^{n(2 n+1)}: n \geq 0\right\}\right.$, $\left.\left\{V^{2 n+1} W^{n(2 n+1)}: n \geq 0\right\}\right]$ and let $\mathscr{P}$ be the set of prime ideals of $D$ which are contractions of height one primes of $K[U, V, W]$ that do not contain $W$. Then the ring $R=D+B$ is its own total quotient ring so, trivially, it is completely integrally closed. But as in the example of Gilmer and Heinzer, both $u w$ and $v w$ are almost integral over $R$, at least they are when considered as elements of $T(R[X]))$. In fact both elements are integral over $R$. For example, written as an element of $T(R[X])$,

$$
u w=\left(u^{10} w^{10} X+u v^{9} w^{10}\right) /\left(u^{9} w^{9} X+v^{9} w^{9}\right) .
$$

Note that this expression makes sense since no prime ideal in $\mathscr{P}$ contains powers of both $U W$ and $V W$. Hence $u w$ satisfies the integrality equation $Z^{9}-u^{9} w^{9}=0$. Similar expressions and equations can be found for any of the products $u^{j} w^{k}$ and $v^{j} w^{k}$ provided $j>0$. Hence the complete integral 
closure of $R$ in $T(R[X])$ can be identified with the ring $R^{\#}=D^{\#}+B$ where $D^{\#}=K\left[\left\{U W^{n}, V W^{n}: n \geq 0\right\}\right]$. As in Example 1.1, the complete integral closure of $R^{\#}$ is the ring $R^{\# \#}=D^{\# \#}+B$ where $D^{\# \#}=K[U, V, W]$. Therefore even though $R$ is completely integrally closed, neither $R[X]$ nor the complete integral closure of $R[X]$ is completely integrally closed. However, $R^{\# \#}[X]$ is completely integrally closed since $K[U, V, W]$ is completely integrally closed.

The example above brings up the following question. If a reduced ring $R$ is completely integrally closed, does the process of taking successive complete integral closures of $R[X]$ terminate in a finite number of steps? On the one hand, examples by Hill [Hi, Theorem 2] and Lantz [Lan, p. 956] show that it is possible to construct infinite chains of domains $D_{1} \subset D_{2} \subset \cdots$ such that for each $j, D_{j+1}$ is the complete integral closure of $D_{j}$. On the other hand, Roitman has recently shown that for domains with acc on divisorial ideals, the process stops in at most two steps [R, Theorem 1.4].

Example 2.6 (cf. [Hu, Example 17]). Let $D$ be a Dedekind domain with a nonprincipal maximal ideal $M=(u, v)$ where neither $(u)$ nor $(v)$ is $M$ primary. Let $\mathscr{P}$ be the set of nonzero prime ideals different from $M$ and form the ring $R=D+B$. Then $R=T(R)$ and $R[X]$ is completely integrally closed since $D$ is completely integrally closed. Also since $M^{-1}$ properly contains $D$, $R \neq Q_{0}(R)$.

The ring in the example above is strongly Prüfer; that is, every finitely generated dense ideal is locally principal. In general, every total quotient ring is Prüfer (every finitely generated regular ideal is invertible), but not necessarily strongly Prüfer. It is easy to see that a Prüfer ring with property $A$ is strongly Prüfer and the example above shows that the converse does not hold. In [D], Dixon put together results in [A2 and AAM] to show that if $R$ is an integrally closed reduced ring and $T(R)$ is strongly Prüfer, then $R[X]$ is integrally closed (see also [Hu, p. 118]).

While it is not the case that $R[X]$ being completely integrally closed implies $T(R)[X]$ is completely integrally closed, the converse does hold (assuming, of course, that $R$ is completely integrally closed). Since $R$ has property $\mathrm{A}$ if and only if $T(R)$ has property A, we see from Corollary 1.8 that if $T(R)$ has property (A) (and hence, is strongly Prüfer), then $R[X]$ is completely integrally closed if and only if $R$ is a completely integrally closed reduced ring. The natural question to ask is whether $T(R)$ being strongly Prüfer is sufficient for $R[X]$ to be completely integrally closed when $R$ is a completely integrally closed reduced ring. We do not know the answer to this question, but our next example shows that $Q_{0}(R)$ need not be strongly Prüfer, and hence it need not have property A.

Example 2.7 (cf. [Hu, Example 18]). Let $D=K[X, Y]$ and let $\mathscr{P}$ be the set of nonzero principal primes of $D$. Then the ring $R=D+B$ is not strongly Prüfer even though $R=T(R)=Q_{0}(R)$

Since $(X, Y)$ is not locally principal, the same thing is true for the dense ideal $(x, y)$ of $R$. Since $D$ is a UFD, the only ideals $A$ of $D$ with $A^{-1} \neq D$ are those contained in principal prime ideals. Thus by repeating the appropriate part of the proof of Theorem 2.2 , we have $\operatorname{Hom}(J, R)=R$ for all finitely generated dense ideals of $R$. Hence $R=Q_{0}(R)$. 


\section{SEMIGROUP RINGS}

Let $S$ be a (nontrivial) torsion-free cancellative (abelian) monoid with quotient group $G$. Let < be a total order on $G$ compatible with the operation. As in the case of the polynomial ring $R[X]$, the results of this section depend upon knowing that since $Q(R)$ is von Neumann, $Q(R)[G]$ is both integrally closed and completely integrally closed.

As in [G2, Chapter 12] we write elements of $R[S]$ essentially as polynomials, specifically for $f(X) \in R[S], f(X)=f_{m} X^{s_{m}}+\cdots+f_{0} X^{s_{0}}$ where $s_{0}<\cdots<s_{m}$.

Theorem 3.1 (cf. [G2, Corollary 12.6]). Let $Q$ be a von Neumann regular ring and let $G$ be a torsion-free (abelian) group. Then $Q[G]$ is completely integrally closed.

Proof. Let $s \in T(Q[G])$ be almost integral over $Q[G]$. Then there is an element $g$ in $Q[G]$ such that $s^{n} g$ is in $Q[G]$ for all $n$. Thus there is a finitely generated subgroup $H$ of $G$ such that $s \in T(Q[H])$ and $g \in Q[H]$. Since $H$ is finitely generated and torsion-free, $H \cong \mathrm{Z}^{n}$ for some $n$ and $Q[H] \cong Q\left[X_{1}, \ldots, X_{n}\right]\left[X_{1}^{-1}, \ldots, X_{n}^{-1}\right]$. Hence by Corollary $1.8, Q[H]$ is completely integrally closed. Therefore $s \in Q[H] \subset Q[G]$ and $Q[G]$ is completely integrally closed.

Recall that the complete integral closure of $S$ is the monoid $S^{*}=\{t \in G$ : for some $s \in S, n t+s \in S$ for all $n \geq 0\}$.

Corllary 3.2. Let $Q$ be a von Neumann regular ring and let $S$ be a torsion-free cancellative monoid. Then $Q\left[S^{*}\right]$ is the complete integral closure of $Q[S]$.

Proof. Let $b \in T(Q[S])$ be almost integral over $Q[S]$ and let $g \in Q[S]$ be such that $b^{n} g \in Q[S]$ for all $n$. Then as in the proof above we may assume that there is a finitely generated subgroup $H$ such that $b \in Q[H]$ and $g \in$ $Q[H] \cap Q[S]$. Hence we may assume that the quotient group $S$ is $\mathbf{Z}^{m}$ for some $m$.

Let $M$ be a prime ideal of $Q$. Then $Q_{M}$ is a field since $Q$ is 0 -dimensional. Thus by [G2,Theroem 12.5] the complete integral closure of $Q_{M}[S]$ is $Q_{M}\left[S^{*}\right]$. Since $Q$ is a reduced ring no coefficient of $b$ is in every prime ideal of $Q$. Thus $b \in Q\left[S^{*}\right]$.

Corollary 3.3. Let $Q$ be a von Neumann regular ring and let $G$ be a torsion-free group. Then $Q[G]$ is integrally closed.

We are now ready to characterize both the complete integral closure and the integral closure $R[S]$. We begin with the complete integral closure.

Theorem 3.4. Let $R$ be a reduced ring and let $S$ be a torsion-free cancellative monoid. Then the complete integral closure of $R[S]$ is the ring $R^{\#}\left[S^{*}\right]$ where $R^{\#}=\lim \{\operatorname{Hom}(J, J): J \in d\}$. In particular, $R[S]$ is completely integrally closed if and only if $R$ is completely integrally closed in $T(R[X])$ and $S$ is completely integrally closed in $G$.

Proof. Since $R^{\#}$ is contained in $T(R[S])$, the elements of $R^{\#}[S]$ are almost integral over $R[S]$ as elements of $T(R[S])$. Also, for each element $t$ of $S^{*}$, $X^{t}$ is almost integral over $R[S]$ since for some $s \in S, X^{n t+s}$ is in $R[S]$. Thus $R^{\#}\left[S^{*}\right]$ is contained in the complete integral closure of $R[S]$. 
Let $b \in T(R[S])$ be almost integral over $R[S]$. By Theorem 3.1, $b$ is an element of $Q(R)[G]$ and hence in $Q(R)[H]$ for some finitely generated subgroup $H$. Using essentially the same technique employed in the proof of Theorem 3.1, it is easy to mimic the proofs of the lemmas in $\S 1$ to show that $b$ is an element of $Q_{0}[H]$. Thus we may assume $G=Z^{n}$ so that $R[S]$ is a subring of $R\left[X_{1}, X_{2}, \ldots, X_{n}\right]\left[X_{1}^{-1}, X_{2}^{-1}, \ldots, X_{n}^{-1}\right] \subset T(R[S])$. Thus by Corollary 2.9, $b \in R^{\#}\left[X_{1}, \ldots, X_{n}\right]\left[X_{1}^{-1}, \ldots, X_{n}^{-1}\right]$ and by Corollary 3.2, $b \in Q(R)\left[S^{*}\right]$. Therefore $b \in R^{\#}\left[S^{*}\right]$ and $R^{\#}\left[S^{*}\right]$ is the complete integral closure of $R[S]$.

Before characterizing the integral closure of $R[S]$, we recall the following result from [Lu2].

Theorem 3.5 (cf. [Lu2, Theorem 3]). Let $R$ be a reduced ring. Then the integral closure of $R[X]$ is the ring $R^{\ell}[X]$ where $R^{\delta}$ is the integral closure of $R$ in $Q_{0}(R)$.

Theorem 3.6. Let $R$ be a reduced ring. Then the integral closure of $R$ in $Q_{0}(R)$ is the ring $R^{\ell}=\underset{\lim }{\longrightarrow}\{\operatorname{Hom}(A, A): A$ is finitely generated dense ideal of $R\}$.

Proof. Let $s \in Q_{0}(R)$ be integral over $R$. Then there is a finitely generated dense ideal $A=\left(a_{0}, a_{1}, \ldots, a_{m}\right)$ such that $s \in \operatorname{Hom}(A, R)$. Moreover we can write $s=\left(b_{m} X^{m}+\cdots+b_{0}\right) /\left(a_{m} X^{m}+\cdots+a_{0}\right)$ where $s a_{k}=b_{k}$ for each $k$. Let $f(Z)=Z^{n+1}+f_{n} Z^{n}+\cdots+f_{0}$ be an equation of integrality of $s$ over $R$ and let $C=c\left(a^{n}\right)+c\left(a^{n-1} b\right)+\cdots+c\left(b^{n}\right)$ where $a=a_{m} X^{m}+\cdots+a_{0}$ and $b=b_{m} X^{m}+\cdots+b_{0}$. We will show that $s \in \operatorname{Hom}(C, C)$. For each $j>0$, we have $s a^{j} b^{n-j}=a^{j-1} b^{n-j+1} \in R[X]$ and $s c\left(a^{j} b^{n-j}\right)=c\left(a^{j-1} b^{n-j+1}\right)$ since $s=b / a$ and $s a_{k}=b_{k}$.

To see that $s c\left(b^{n}\right) \subset C$ we need to use the integrality equation. Write $s^{n+1}=-\left(f_{n} s^{n}+\cdots+f_{0}\right)$. Replacing each $s^{j}$ by $b^{j} / a^{j}$ we get $s\left(b^{n} / a^{n}\right)=$ $-\left(f_{n} b^{n}+f_{n-1} b^{n-1} a+\cdots+f_{0} a^{n}\right) / a^{n}$. Hence $s b^{n}=\left(f_{n} b^{n}+\cdots+f_{0} a^{n}\right)$. Whence, $s c\left(b^{n}\right) \subset C$ and $s C \subset C$ as desired. Thus $R^{\&}$ contains the integral closure of $R$ in $Q_{0}$.

Conversely, since $Q_{0}(R) \subset T(R[X])$, no nonzero element of $Q_{0}(R)$ can annihilate a finitely generated dense ideal of $R$. Hence as in the domain case, if $s A \subset A$ for some finitely generated dense ideal of $R$, Cramer's Rule implies $S$ is integral over $R$.

Theorem 3.7. Let $R$ be a reduced ring and let $S$ be a torsion-free cancellative monoid. Then $R^{\ell}\left[S^{\prime}\right]$ is the integral closure of $R[S]$.

Proof. By Corollary 3.3 the integral closure of $R[S]$ is contained in $Q(R)[G]$. As with complete integral closure, the integral closure of $Q(R)[S]$ is $Q(R)\left[S^{\prime}\right]$ since $Q(R)_{M}$ is a field (cf. [G2, Corollary 12.11]). Hence the integral closure of $R[S]$ is a subring of $Q(R)\left[S^{\prime}\right]$ and contains $R^{\delta}\left[S^{\prime}\right]$.

Since $R^{\ell}\left[S^{\prime}\right]=R^{\ell}[G] \cap Q(R)\left[S^{\prime}\right]$, to complete the proof all we need do is to show that $R^{\&}[G]$ is integrally closed. As in the proof of Theorem 3.4 we may assume that $G$ is finitely generated and hence $G=\mathbf{Z}^{m}$ for some $m$. For a single indeterminate $X$, the integral closure of $R[X]$ is $R^{b}[X]$. Since polynomial rings have property $\mathrm{A}, R^{\delta}\left[X_{1}, \ldots, X_{n}\right]$ is integrally closed by [A1, Theorem 3.2]. Thus $R^{\ell}\left[X_{1}, \ldots, X_{n}\right]\left[X_{1}^{-1}, \ldots, X_{n}^{-1}\right]$ is integrally closed and therefore so is $R^{\mathscr{Q}}[G]$. 


\section{REFERENCES}

[A1] T. Akiba, Integrally-closedness of polynomial rings, Japan J. Math. 6 (1980), 67-75.

[A2] - On the normality of $R(X), J$. Math. Kyoto Univ. 20 (1980), 749-752.

[AAM] D. D. Anderson, D. F. Anderson and R. Markanda, The rings $R(X)$ and $R\langle X\rangle$, J. Algebra 95 (1985), 96-115.

[BCM] J. Brewer, D. L. Costa and K. McCrimmon, Seminormality and root closure in polynomial rings and algebraic curves, J. Algebra 58 (1979), 217-226.

[C] L. Claborn, Every abelian group is a class group, Pacific J. Math. 18 (1966), 219-222.

[D] A. Dixon, A polynomial ring localization: $R\{X\}$, Dissertation, Univ. of Missouri-Columbia, 1987.

[G1] R. Gilmer, Multiplicative ideal th.ory, Dekker, New York, 1972.

[G2] _ Commutative semigroup rings, The Chicago Univ. Press, Chicago, 1984.

[GH] R. Gilmer and W. Heinzer, On the complete integral closure of an integral domain, J. Austral. Math. Soc. 6 (1966), 351-361.

[Hi] P. Hill, On the complete integral closure of a domain, Proc. Amer. Math. Soc. 36 (1972), 26-30.

[Hu] J. Huckaba, Commutative rings with zero divisors, Dekker, New York, 1988.

[Lam] J. Lambek, Rings and modules, Chelsea, New York, 1986.

[Lan] D. Lantz, Finite Krull dimension, complete integral closure and GCD-domains, Comm. Algebra 3 (1975), 951-988.

[Lu1] T. Lucas, Characterizing when $R[X]$ is integrally closed, Proc. Amer. Math. Soc. 105 (1989), 861-867.

[Lu2] Characterizing when $R[X]$ is integrally closed. II, J. Pure Appl. Algebra 61 (1989), 49-52.

[Lu3] _ Root closure and $R[X]$, Comm. Algebra 17 (1989), 2393-2414.

[Q] Y. Quentel, Sur la compacité du spectre minimal d'un anneau, Bull. Soc. Math. France 99 (1971), 265-272.

[R] M. Roitman, On the complete integral closure of a Mori domain, preprint.

Department of Mathematics, University of North Carolina at Charlotte, CharLOTTE, North CARolina 28223 